\title{
WHAT FUTURE FOR RADIOCARBON?
}

\author{
E M Scott $・$ D D Harkness \\ Department of Statistics, University of Glasgow and Scottish Universities Environmental Research Centre
}

\begin{abstract}
In this short article, we summarize some milestones in the 50 -yr-long development of natural ${ }^{14} \mathrm{C}$ measurement. In the light of this appraisal we presume to hazard some personal opinions and forecasts as to where best opportunities might lie for future gains from the continued investment in applied ${ }^{14} \mathrm{C}$ science. The technique and the journal are one and the same in this regard.
\end{abstract}

\section{INTRODUCTION}

In the half century that has elapsed since the first reported measurements of naturally occurring ${ }^{14} \mathrm{C}$ the breadth of applied carbon isotope science has grown almost exponentially; reaching out from archaeology through oceanography, Quaternary science, geography and indeed to embrace most if not all of those disciplines that fall within the wide spectrum of "Earth science". At the root of this opportunity for academic contribution is of course the ubiquitous role of elemental carbon in the very biogeochemistry of life itself.

An early recognition of the importance of the radiocarbon dating method is perhaps best evidenced by the rapid expansion in the number of laboratories prepared to accept the considerable technical challenge posed by the need for the quantitative and reproducible detection of ${ }^{14} \mathrm{C}$ in various natural materials. This via radiometric counting of weak energy beta emissions occurring at ultra-low specific activities. Nevertheless, over the first two decades or so the number of active "radiocarbon dating laboratories" increased from single figures to over 100 which were by then distributed worldwide. An ongoing expansion in scientific demand served to drive appropriate technological developments and/or innovative approaches to isotope analyses per se. For example, those pioneer laboratories initially dependent on the preparation and monitoring of solid carbon sources were soon retooling to benefit from the advantages afforded by the newer gas proportional or liquid scintillation counting techniques.

A parallel demand for measurements on an ever more diverse range of naturally occurring materials and often in conjunction with smaller samples came from a varied congregation of "user scientists" who were keen to expound new hypotheses and to ask the inevitable searching questions. As a general rule, the "radiocarbon provider community" responded accordingly to this perceived need for interdisciplinary collaboration. It did so mainly by the development and delivery of significant technical improvements and/or new analytical procedures. A major breakthrough in the technological challenge came with the advent of ${ }^{14} \mathrm{C}$ AMS (accelerator mass spectrometry). Direct measurement of the relative isotopic abundance of natural ${ }^{14} \mathrm{C}$ was no longer constrained by radiometric counting i.e., the need to achieve an acceptable analytical precision at specific activities of less than $12 \mathrm{dpm} /$ gmC. Populations of ${ }^{14} \mathrm{C}$ atoms could now be monitored without having to wait around for individuals to pop off. The immediate advantages of much smaller sample size and significantly less experimental lead-times has opened up a veritable Pandora's box of scientific opportunity for the applied science. Albeit there is disappointment in some quarters that, as experience has shown, the expertise of high energy physics has not been able to extend the age range of the radiocarbon chronology or for that matter to improve on the dating precision achieved routinely by established radiometric counting labs. Nature itself retains ultimate control over these aspects of the applied science by virtue of setting the relative isotope abundance and radioactive half-life of ${ }^{14} \mathrm{C}$. 
Looking back over the half century of radiocarbon exploitation a salient feature is the success story penned by the constant interplay and mutual stimulation of user demand and provider response. However, this observation poses an obvious question over the continuing evolution of radiocarbon science viz.,

Are there still technical developments to come in natural ${ }^{14} \mathrm{C}$ measurement which will seed and/or satisfy new areas of scientific enquiry or has the applied science reached its apex?

Our response here must be an emphatic no! The road ahead for natural ${ }^{14} \mathrm{C}$ measurement would seem to lead through many fertile fields ranging from those unfinished tasks in reconstructing the last 50,000 years or so of Earth history, through the ever more evident need for improved management of our planetary resources.

We hope our attempt at a predicted route map, although entirely personal will nevertheless resonate with some of our readers.

\section{Some Markers in the History of Applied ${ }^{14} \mathrm{C}$ Science}

The scientific value of radiocarbon stems from the remarkable coincidence of several natural phenomena. First and foremost is the very fact that the fundamental element of life (carbon) occurs in nature with a radioactive isotope. Moreover, the characteristic half-life of this radioactive label is set conveniently within a range commensurate with the significant progress of human civilization. The geochemistry also appears as having been similarly contrived to set the challenge of radiocarbon dating viz., the continual cosmogenic production of ${ }^{14} \mathrm{C}$ allied to its virtually immediate atmospheric homogeneity and direct biogenic availability in the gaseous phase. Of course the natural system is not ideal in all respects. We would all like to experience a greater ${ }^{14} \mathrm{C}$ natural abundance than the somewhat parsimonious $10^{-12}$, and while archaeologists might be better served by a lesser half-life, nature's determination of $5730 \mathrm{yr}$ tends to be a wee bit short for geological preference.

Archaeology provided the original driving force for development of the dating method since archaeologists were then in sore need of a secure time-scale on which to base and compare theories of succession and/or contemporaneity which were otherwise almost entirely dependent on assumed similarities among the physical debris of pre-history. Although archaeology remains prominent in the chronology's user group, scientific interest in the radiocarbon clock has seen a gradual shift to the reconstruction of natural rather than cultural episodes and pertaining to the past 50,000 years of Earth history. This change in emphasis for the applied science reflects the growing tendency to harmonize and integrate man into the carbon cycle; his interaction with the environment forces changes, but in the past, natural environmental changes have also forced changes in customs, and caused populations to migrate and /or adopt new habits.

Among typical user groups there has been a growing awareness of the fundamental importance of sampling integrity and in context of the true association between the measured ${ }^{14} \mathrm{C}$ age of the selected material and the event that it assumed to date. The more ready availability of age measurements and the removal of size restrictions via ${ }^{14} \mathrm{C}$ AMS has encouraged a move away from dependence on dating single samples pertaining to a recognized event. It is now common to encounter 1) the multiple dating of discrete physical or chemical components isolated from a given sample, and 2) the interpretation of series and /or sequences of related dates using complex mathematical methods.

For the experimental chronologist there still exists the incentive to improve on both the analytical quality and user confidence in the objective interpretation of results. High on the agenda for the past 30 years or so has been the concerted effort by several specialist laboratories to chart accurately 
those now well recognized wrinkles in the conventional ${ }^{14} \mathrm{C}$ time-scale that derive from past changes in natural ${ }^{14} \mathrm{C}$ production and/or transient perturbations in the dynamics of the natural carbon cycle. This high-precision calibration of the conventional radiocarbon time-scale against the absolute and continuous calendar provided by dendrochronology is painstaking work which continues even today (see the INTCAL 98). Furthermore, the need for extension of the ${ }^{14} \mathrm{C}$ calibration curve beyond the availability of suitable tree-ring sequences has encouraged a focus on more recent research which seeks to exploit the independent timers inherent in the growth structure of corals and/or varved sediments and also explores the relationship to other naturally occurring radionuclides (a comprehensive account of progress in these areas will feature in a forthcoming issue of this journal).

A concern for improved user confidence has stemmed from the continuing need to emphasize that natural radiocarbon measurement and in particular its interpretation as years past is not an absolute tool. It must be recognized that there is an inherent level of uncertainty in all results which depend on quantifying trace amounts of a radioactive isotope. It becomes incumbent on the measuring laboratory to ensure that its user scientists have an appropriate appreciation of both the accuracy and the precision that can be assigned for the reported conventional age and/or radiometric enrichment (e.g., pMC) values. In this context it is very satisfying to note that the vast majority of operational radiocarbon laboratories now address this essential aspect of customer care by their open participation in previous and ongoing intercalibration studies.

Most recently in terms of the novel use of low-level ${ }^{14} \mathrm{C}$ measurement is the exploitation of AMS technology in biomedical research. In particular, the quantitative tracing of processes that are essential to human metabolism e.g., nutritional effects, dietary studies and drug kinetics. While such work is to be commended and encouraged as being both intellectually exciting and socially satisfying it falls more in the category of the laboratory contained tracer study, perhaps then best regarded as a positive spin-off from the art of natural radiocarbon measurement.

\section{Where are We Now?}

What is the state-of-the-community message after neigh on fifty years of technical development and practical experience in natural ${ }^{14} \mathrm{C}$ measurement? In essence:

We can employ a universal chronometer covering the past 50,000 years of Earth history. However, as discussed previously, nature seems to have set this as the upper practical limit by virtue of half-life and natural abundance. The need for a total avoidance of trace modern carbon in both field sampling and laboratory based procedures (the inevitable background question) is a comparable analytical constraint and irrespective of technical sophistication.

We can achieve as a matter of routine an analytical precision of better than $0.5 \%$ and do so for samples that provide somewhat less than $1 \mathrm{mg}$ carbon. Thus ages pertaining to the last 12,000 years or so are reproduced with an analytical confidence of a few decades. Given sufficient sample size, the high-precision technology developed for dendrochronological calibration can achieve \pm 15 years or less where this is justified by sample integrity. Alternatively, under favorable conditions, a virtually absolute dating option is available via allied mathematical fixes such as wiggle matching to the master calibration curve.

We can, as already mentioned, achieve a ${ }^{14} \mathrm{C}$ measurement from even microgram amounts of carbon. However, it must be questioned whether there is a danger that, for the unwary user, this could represent a step too far in technological capability? Small may be beautiful and exciting but in many applied contexts there must surely be a limit below which the ultra-small carbon sample cannot be assumed to truly represent the particular event or natural process. 
Perhaps present day levels of attainment in the technical aspects of natural ${ }^{14} \mathrm{C}$ measurement should be accepted as being at or close to our ultimate objective.

\section{Where are We Likely to Go?}

The short answer is, "still a long way". However, it seems to us inevitable that the intellectual challenge of natural ${ }^{14} \mathrm{C}$ measurement will shift even more from technological improvement towards the realms of scientific application. There would seem to be more than enough opportunity for research satisfaction here and particularly so if the already obvious trends for amalgamation with other chronological methods and/or the exploitation of radiocarbon's counterpart cosmogenic radionuclides continue.

A summary overview of some applied ${ }^{14} \mathrm{C}$ research opportunities can be set most conveniently in context of the natural carbon cycle since this concept offers a convenient framework in which to sketch the complex bio-geochemical interplays that exist within and among the atmosphere, biosphere, and ocean.

The atmosphere is of course the area of cosmogenic production and as such it will continue to be a primary focus for the study of those geophysical and extraterrestrial factors that have determined past variations in the production of radiocarbon and/or the other radionuclide byproducts of the primary cosmic flux.

More immediate interest, and in relation to management of the global environment, centres on isotopic tracing and temporal quantification of the anthropogenic sources for those carbonaceous gases $\left(\mathrm{CO}_{2}\right.$ and $\left.\mathrm{CH}_{4}\right)$ liable to contribute to climate change, and in determining the nature and ultimate response capacity of their natural sinks. Radiocarbon must stand ready to input unambiguous information and answers for future political debate centred on the "Kyoto Question". Likewise in context of insidious pollution, is the need to monitor the significant and long-term radiation dose rate being delivered from ${ }^{14} \mathrm{C}$ produced during the nuclear weapons testing programs together with localized emissions from our continuing dependence on the nuclear fuel cycle.

Since there is a direct and virtually instantaneous coupling of atmospheric carbon to the tissue of living plants, the well mixed $\mathrm{CO}_{2}$ reservoir is effectively the primary source for all biologically recorded variations in ${ }^{14} \mathrm{C}$ concentration whether this is a consequence of natural forces or human perturbation e.g., the "Suess" and "bomb" effects.

The terrestrial biosphere is considered here as comprising all carbon contained in those chemical structures that can be ascribed to an organism that has lived at sometime during the range of the radiocarbon time-scale i.e., the past 50,000 years. It of course includes all plants and animals that are presently alive on land and in freshwater systems.

This carbon reservoir affords by far the greatest range of opportunity for improving established research themes and the development of novel approaches. Two broad categories exist viz., 1) the ordering and/or reconstruction of past events, and 2) quantitative tracing of the modern pathways and/or fluxes of environmental carbon.

In the first category we have of course radiocarbon dating support for archaeology where continuing emphasis must surely be towards improved confidence in the conversion of conventional ages to calendar dates. Confident calibration is essential to meet a changing culture within archaeological research in which understanding the relationships between cultures and cultural change, and delineating sequences of events or activities takes priority over simple dating of a proxy sample. 
We have already touched on the incentive to extend the radiocarbon calibration curve beyond the limit of direct dendrochronological comparison. The obvious call here is for continuing research and development collaboration between ${ }^{14} \mathrm{C}$ practitioners and their counterpart scientists with expertise in appropriate chronological comparators e.g., U/Th dating of corals, Ar/Ar dating, TL and/or OSL techniques and the recovery and counting of varve and ice-core sequences.

There is also a reverse angled viewpoint concerning the importance of the calibration data set. Past temporal changes in natural ${ }^{14} \mathrm{C}$ concentration provide a unique index for comparison of the global synchroneity, rates of change and possible driving mechanisms that obtain for those episodes of major climate change that are variously recorded in many sedimentary archives. A prime example here concerns interpretation of the complex patterns associated with the last transition from full glacial to temperate conditions around the north Atlantic margins (see Lowe and Walker in this issue). Such exercises in environmental reconstruction call increasingly for the development and application of appropriate expertise in the identification and recovery of the most representative components within the bulk organic matrix and in relation to a diversity of host deposits e.g., lake sediments, peat accumulations, paleosols, loess deposits, ice-cores, etc. A particularly challenging and potentially rewarding approach would seem to be with prior compound specific isolation and geochemical definition of the measured organic materials.

Anthropogenic disturbance of the near steady-state distribution of natural radiocarbon viz., via emissions of fossil fuel derived $\mathrm{CO}_{2}$ (Suess effect), injection of the "bomb ${ }^{14} \mathrm{C}$ " spike and massive deforestation programs, still affords unique but transient opportunities to trace carbon transfer rates with an otherwise impossible degree of temporal resolution. The immediate exploitation of the "bomb pulse" to quantify atmospheric mixing patterns, air-to-ocean exchange parameters, and the dynamics of oceanic circulation are well documented. However, the continuing distribution of this excess of artificial ${ }^{14} \mathrm{C}$ through vegetation and soils is still useful for evaluation of the associated organic carbon dynamics. This via the construction and testing of models of carbon flux that can be applied to ensure best practice in the future management of agricultural and forest ecosystems.

Although not all of the carbon contained in groundwater can be ascribed to a biological source it is appropriate to feature the importance of natural ${ }^{14} \mathrm{C}$ measurement in the management of aquifer resources in context of terrestrial deposits. This highly specialized field presents a considerable but entirely satisfying challenge for isotope geochemistry (see review by Geyh in this issue). There can be no doubt that the social and economic importance of adequate water supply is sufficient stimulation for continued research and development effort geared to improve management options.

The World's oceans contain and cover the largest reservoir by far of chemically active carbon. This inventory has been the subject of intensive and productive ${ }^{14} \mathrm{C}$ based research over several decades. Included here has been the charting of circulation patterns, the determination of mixing rates and the dynamics of deep-water formation which was in turn aided by the net input and subsequent distribution of "bomb ${ }^{14} \mathrm{C}$ " tracer. An important outcome of that work has been an appreciation of the paramount role of oceanic mixing patterns and their associated heat transfer capacities in determining the timing and extent of past climate change. Such evidence provides strong justification for continued research focused on the complex carbon isotope geochemistry, both inorganic and organic pools (DIC/DOC/PIC/POC), that characterize discrete water masses. Likewise, the history of past changes in ocean status within the regional environment is retained in the stratified seabed sediments. 


\section{CONCLUSION}

In their foreword to the volume published to mark 40 years of radiocarbon science ${ }^{1}$ editors $\mathrm{R} E$ Taylor, Austin Long, and Renee Kra (1992) highlighted a citation made in support of Willard Libby's nomination as Nobel laureate viz.,

Seldom has a single discovery in chemistry had such an impact on the thinking of so many fields of human endeavour. Seldom has a single discovery generated such wide public interest.

After a further decade of extremely interesting and productive developments in applied ${ }^{14} \mathrm{C}$ measurement the sentiment is equally if not even more appropriate today.

The international "radiocarbon family" continues to grow and prosper. At the firm foundation of this success is the scientific kinship forged through interdisciplinary stimulation and allied to an attitude of unstinting collaboration. The necessary home for this success has been provided by the journal Radiocarbon and in practice via its marriage of sound editorial policy with the highest levels of professional publishing practice.

Here's to the next 50 years!

\section{REFERENCE}

Taylor RE, Long A, Kra RS, editors. 1992. Radiocarbon after four decades: an interdisciplinary perspective. New York: Springer-Verlag Inc. 